\title{
Evaluación del impacto en la morbimortalidad con la aplicación de un protocolo en sangrado mayor en el Hospital Universitario, Hernando Moncaleano Perdomo de Neiva
}

\author{
Evaluation of the effect on morbidity and mortality of the application of a \\ protocol in cases of major bleeding in Hospital Universitario Hernando \\ Moncaleano, Neiva
}

Daniel Rivera Tocancipá', Carlos Andrés Rivera Ortiz², Miguel Francisco Sandoval Cabrera ${ }^{3}$

\begin{abstract}
Resumen
Introducción: El sangrado mayor es frecuente en salas de cirugía y causa el $40 \%$ de las muertes por trauma. Su diagnóstico y tratamiento adecuado por anestesiólogos, es prioritario para la sobrevida. No hay datos en el Hospital Universitario de Neiva sobre la morbimortalidad en este escenario ni existe un protocolo de manejo estandarizado.

Objetivo: Determinar la morbimortalidad a las 24 horas y mortalidad a 30 días, en pacientes mayores de 15 años con diagnóstico de sangrado mayor en salas de cirugía, aplicando un protocolo estándar de manejo. Material y Métodos: Se diseñó un estudio cuasi experimental para pacientes con sangrado mayor. La cohorte prospectiva recibió protocolo estándar (PE) de manejo. Se determinó el valor de lactato sérico, base exceso y mortalidad a las 24 horas y 30 días, comparándola con la cohorte histórica tomada de los registros de la Unidad de Cuidados Intensivos. Resultados: De 63 pacientes, 26(41.9\%) fueron controles retrospectivos sin protocolo de manejo, $13(21.1 \%)$ recibieron protocolo completo y 24 (38\%) recibieron protocolo incompleto. Con protocolo la mortalidad proyectada para las 24 horas bajó de $48 / 1000$ a $32 / 1000$. A los 30 días sin protocolo la tasa de mortalidad fue de $129 / 1000$, con protocolo incompleto de 48/1000 y con protocolo completo de 32/1000. El lactato sérico disminuyó y la base exceso aumentó cuando se aplicó el protocolo de manejo. Conclusiones: La aplicación de un protocolo de manejo en pacientes que cursen con sangrado mayor, disminuye la mortalidad a las 24 horas y 30 días del evento desencadenante en un $70 \%$.
\end{abstract}

Palabras clave: Sangrado Mayor; Trauma; Anestesia General; Reanimación.

\section{Abstract}

Introduction: Major bleeding is common in the operating room and is the cause of $40 \%$ of trauma deaths. Diagnosis and appropriate treatment from anaesthesiologists are crucial for survival. Hospital

1. MD. Anestesiólogo con entrenamiento en trasplante hepático y Anestesia Pediátrica de Alta complejidad. Docente Postgrado Anestesiología y Reanimación Universidad Surcolombiana de Neiva.

2. MD. Anestesiólogo. Universidad Surcolombiana. Neiva.

3. MD. Residente I. Anestesiología y Reanimación. Universidad Surcolombiana de Neiva. Correspondencia: Daniel Rivera Tocancipá. Correo electrónico: riverato@hotmail.com 
R.F.S Revista Facultad de Salud

Enero-Junio de 2015;7(1): 17-23
Evaluación del impacto en la morbimortalidad con la aplicación de un protocolo en sangrado mayor/Daniel Rivera T., Carlos A. Rivera O., Miguel F. Sandoval C.

Universitario, Neiva, holds no data about morbidity and mortality in this particular scenario, nor is there a standardised protocol to follow if major bleeding occurs. Objective: Determine morbidity and mortality in 24 hours and mortality in 30 days in patients over the age of 15 years diagnosed with major bleeding in the operating room, implementing a standard protocol. Equipment and Methods: A quasi-experimental study for patients with major bleeding was designed. For the prospective cohort, standard protocol was implemented. The serum lactate level, base excess and mortality in 24 hours and 30 days were recorded and then compared with the historic cohort taken from the records of the Intensive Care Unit. Results: Of 63 patients, 26 (41.9\%) were controlled without the need for protocol, $13(21.1 \%)$ received the complete protocol and an incomplete protocol was implemented in $24(38 \%)$ cases. Where the protocol was implemented, the projected mortality in 24 hours fell from 48/1000 to $32 / 1000$. In 30 days, the mortality rate was $129 / 1000$ where the protocol was not implemented, $48 / 1000$ where an incomplete protocol was implemented and 32/1000 where the complete protocol was implemented. When the protocol was applied, the serum lactate level reduced and the base excess increased. Conclusions: Applying the protocol in patients with major bleeding reduces mortality in 24 hours and 30 days after the triggering event by $70 \%$.

Key words: Bleeding, trauma, general anaesthesia, resuscitation.

\section{Introducción}

La hemorragia no controlada produce aproximadamente el $40 \%$ de las muertes traumáticas ${ }^{1,2}$. El tratamiento requiere instaurar acciones inmediatas para controlar el sangrado y restaurar la perfusión tisular ${ }^{3}$.

Se puede requerir una transfusión masiva ${ }^{4}$ ante la presencia de sangrado mayor. La utilización inadecuada de hemoderivados, la misma trasfusión, el desconocimiento de la fisiopatología y el no monitorizar la coagulación ${ }^{5}$, puede llevar a una situación crítica al organismo induciendo acidosis, hipotermia y coagulopatía ${ }^{6-8}$ llamadas la "triada de la muerte".

Este estudio pretende correlacionar el valor del lactato sérico y base exceso como medidores de morbilidad a las 24 horas, así como la mortalidad a las 24 horas y 30 días postrauma, con y sin la aplicación de un protocolo estandarizado $(\mathrm{PE})$, entendiéndose por trauma la lesión inicial que desencadena el sangrado mayor. El PE incluye la monitorización y tratamiento de los niveles de calcio, base exceso, fibrinógeno, temperatura y coagulación mediante el PT y PTT a cambio de la ideal tromboelastografía ${ }^{9,10}$ con la cual no contamos al momento del estudio e incluyó las transfusiones de glóbulos rojos empaquetados, plaquetas y plasma fresco congelado ${ }^{11}$ en relación $1: 1: 1^{12}$.

Las variables medidas pretenden obtener una medida global e indirecta del débito de oxígeno, perfusión tisular, coagulopatía y severidad del choque hemorrágico. Además el lactato sérico predice sobrevida y pronóstico ${ }^{13}$. El desenlace principal es la tasa de mortalidad a 30 días por grupo de comparación. Los resultados permitirán conocer la importancia e impacto de la aplicación de un protocolo de manejo para el paciente con sangrado mayor.

\section{Material y métodos}

La hipótesis fue que la aplicación de un PE para pacientes mayores de 15 años, que cursaran con sangrado mayor, redu- ce la morbilidad reflejada por los niveles de lactato, calcio, base exceso, fibrinógeno y temperatura, medidos a las a las 24 horas y reduce la mortalidad a las 24 horas y 30 días del evento precipitante.

Se realizó un estudio cuasi-experimental de antes y después en el que se compararon los datos de pacientes que cursaron con sangrado mayor de una cohorte prospectiva recolectada desde el 1 de Julio de 2011 hasta 31 Diciembre de 2012, y otra cohorte histórica tomada antes de la implementación del PE desde el 1 de junio de 2010 hasta el 31 de mayo de 2011 obtenidos de los libros de registro de salas cirugía, el libro de ingresos a la Unidad de Cuidados Intensivos y el Libro de Ingreso a Urgencias. A la cohorte prospectiva de intervención se le aplicó el \# Protocolo Estandarizado" (PE) para sangrado mayor midiendo lactato sérico, calcio sérico, fibrinógeno, base exceso y temperatura, como medidas objetivas de la reanimación y reflejo de la "morbilidad" del paciente, tanto al ingreso a quirófanos como a las 24 horas del trauma. Se determinó la mortalidad a las 24 horas y a los 30 días. El estudio se realizó en el servicio de Salas de Cirugía del Hospital Universitario de Neiva, institución de tercer y cuarto nivel de atención.

Se registró la mortalidad a las 24 horas y con seguimiento telefónico o intrainstitucional, la mortalidad a los 30 días.

No se realizó tamaño muestral pues fueron incluídos todos los pacientes durante el periodo del estudio. Una vez reunidos todos los datos de los pacientes recolectados, se realizó el análisis estadístico con el programa Statistical Package for the Social Sciences (SPSS versión 15), de licencia gratuita.

Tratamiento Estadístico: para tratar las variables sociodemográficas y clínicas como lactato sérico y hemoderivados se realizó análisis descriptivo en tablas de frecuencia y se calculó el nivel de significancia para los tres grupos de estudio. Para tratar los datos de cada uno de los grupos formados se optó por la metodología de análisis correlacional intragrupo e intergrupo de acuerdo a las 
variables clínicas y guiado por el desenlace mortalidad a las 24 horas y 30 días.

\section{Criterios de Inclusión:}

- Ser mayor de 15 años.

- Cumplir con criterios de sangrado mayor y que ingrese a salas de cirugía para tratamiento quirúrgico independientemente de la etiología del sangrado. Se definió "Sangrado Mayor" según las Guías de Manejo para Sangrado Mayor del Comité Británico en $2008^{14}$ : pérdida total de la volemia en 24 horas; la pérdida del $50 \%$ de la volemia en menos de 3 horas o una tasa de hemorragia mayor a $150 \mathrm{ml} / \mathrm{min}$. Si no era posible cuantificar la hemorragia se acogía la clasificación de la American College of Surgeons Advanced Trauma Life Support Classification of Haemorrhage Severity ${ }^{15}$, y pacientes en estadío III o IV ingresaron al estudio.

\section{Criterios de Exclusión:}

- Tener antecedentes de discrasia sanguínea.

- Llevar más de 24 horas posterior al evento desencadenante.

- Tener clínica de muerte cerebral.

- Haber recibido hemoderivados sin relación plasma fresco congelado y glóbulos rojos empaquetados 1:1.

- Rechazo del paciente o acudiente a la administración de hemoderivados o a la implementación del protocolo. Rechazo del PE por parte del anestesiólogo del caso.

Protocolo Estandarizado (PE): Se basó en los siguientes 6 puntos:

1. Monitoria: La básica intraoperatoria incluyendo temperatura orofaríngea. Objetivo mínimo de $35^{\circ} \mathrm{C}$, calentando los líquidos endovenosos y con mantas térmicas $^{16}$. Garantizar mínimo dos accesos venosos periféricos con catéteres 16 o 18 Gauge, monitoria invasiva de presión arterial y acceso venoso central para monitoria de presión venosa central (PVC) y administración de agentes vasopresores e inotrópicos.

2. Líquidos endovenosos: Se administra solución salina normal (SSN) o Lactato Ringer hasta $3000 \mathrm{ml}$. Si requiere aumentar el volumen intravascular se continúa con coloides a razón de 20 a 30 cc/kg máximo.

3. Hemoderivados: Iniciar transfusión con relación Glóbulos Rojos Empaquetados, Plaquetas y Plasma 1:1:17 hasta completar 6 unidades de cada uno ${ }^{18,19}$. De ser necesario se repite el esquema.

4. Coagulación: Medición de PT, PTT, INR y fibrinógeno. Objetivo, mantener los valores en normalidad y el fibrinógeno mayor a $1 \mathrm{~g} / \mathrm{dL}$.

5. Gases Arteriales: Toma de gases arteriales al ingreso, en el intraoperatorio, al egreso de cirugía y a las 24 horas postrauma ${ }^{20}$, documentando la base exceso cuyo objetivo es mantenerlo mayor a $-6 \mathrm{mEq} / \mathrm{L}^{21}$, lactato sérico ${ }^{22}$ inferior a $4 \mathrm{mEq} / \mathrm{L}$ y calcio sérico ${ }^{23}$ mayor a $1,2 \mathrm{mmol} / \mathrm{L}^{24}$.

6. Antifibrinolisis: Administra ácido tranexámico, bolo de $10 \mathrm{mg} / \mathrm{kg}$ y continúa infusión $1 \mathrm{mg} / \mathrm{kg} / \mathrm{hora}^{25}$.

\section{Resultados}

Se recolectaron 63 pacientes distribuidos en tres grupos: el primero fue la cohorte histórica, quienes no recibieron ningún tipo de intervención con la intención del PE; el segundo y tercer grupo correspondió a los pacientes reclutados de manera prospectiva a quienes se les aplicó el PE completo o incompleto; este último grupo (aplicación incompleta del PE) fue armado durante el análisis debido a que la adherencia al protocolo no fue del $100 \%$. (Tabla 1).

La distribución por género y edad no presenta diferencias estadísticamente significativas entre los grupos (Tabla 1).

Cuando se analiza los tres grupos en función de la mortalidad, se encuentra que en el grupo protocolo completo el $15.4 \%$ fallecen a las 24 horas sin aumento de la mortalidad a los 30 días. En el grupo protocolo incompleta el $8.7 \%$ de los pacientes fallecieron a las 24 horas, con un posterior aumento de la mortalidad hasta el 13\% a 30 días. Sin embargo, no se encuentran diferencias estadísticamente significativas: $\mathrm{r}=0.363 ; \mathrm{P}=0.609$. En la cohorte histórica que no aplicó el $\mathrm{PE}$ el $11.5 \%$ de los pacientes fallecieron a las 24 horas, con un aumento a $30.8 \%$ de fallecimientos a 30 días. Este aumento de mortalidad de 24 horas a 30 días por grupo, está resaltado con flecha roja en la figura 1. La figura 2 muestra la tasa de mortalidad proyectada por 1000 .

La cohorte histórica presentó los niveles más altos de lactato sérico a 24 horas respecto al ingreso, comparado con el grupo protocolo completo y protocolo incompleto, con diferencias estadísticamente significativas $(\mathrm{p}=$ 0,047) (Tabla 2). La cohorte histórica presentó los niveles más negativos de base exceso a 24 horas, comparado con el grupo protocolo completo y protocolo incompleto, con diferencias estadísticamente significativas $(\mathrm{p}=0,0001)$. (Tabla 2).

La tasa de trasfusión para glóbulos rojos empaquetados fue mínimo de una unidad, máximo 6 unidades con una media de 3,6389 unidades de glóbulos rojos para cada paciente. En cuanto a las unidades de plasma fresco congelado se encontró que la tasa de transfusión fue de mínimo 3 unidades, máximo 8 unidades, con una media de 4,6786 unidades de plasma fresco congelado por cada paciente (Tabla 3).

$\mathrm{Al}$ analizar las plaquetas trasfundidas se encontró que la tasa de transfusión mínima fue de 3 unidades de plaquetas, máximo 6 unidades de plaquetas con una media de 4,1429 unidades de plaquetas por cada paciente (Tabla 3 ).

La trasfusión específica para el grupo de protocolo completo mostró para glóbulos rojos empaquetados una media de 4 unidades, 4,5 unidades para plasma fresco congelado y 4,1 unidades para plaquetas, por paciente. 
R.F.S Revista Facultad de Salud

Enero-Junio de 2015;7(1): 17-23
Evaluación del impacto en la morbimortalidad con la aplicación de un protocolo en sangrado mayor/Daniel Rivera T., Carlos A. Rivera O., Miguel F. Sandoval C.

Tabla 1. Distribución por género - edad y causas de sangrado mayor.

\begin{tabular}{lccc}
\hline Variables No. (\%) & $\begin{array}{c}\text { Protocolo } \\
\text { Completo }\end{array}$ & $\begin{array}{c}\text { Protocolo } \\
\text { Incompleto }\end{array}$ & Control \\
\hline Distribución de grupos & $13(22)$ & $24(38)$ & $26(42)$ \\
\hline $\begin{array}{l}\text { Distribución por géneros } \\
\text { Hombre }\end{array}$ & $20(83)$ & $18(69)$ & $11(85)$ \\
Mujer & $2(15)$ & $4(17)$ & $8(31)$ \\
\hline Distribución por género y edad & & & 29,6 \\
\hline $\begin{array}{l}\text { Hombre } \\
\text { Edad media en años }\end{array}$ & 31,6 & 32,5 & 15,13 \\
$\begin{array}{l}\text { Desviación estándar } \\
\text { Mujer }\end{array}$ & 14,43 & 15,4 & \\
Edad media en años & 31,4 & 31,1 & 8,05 \\
Desviación estándar & 19,42 & 31 & \\
\hline
\end{tabular}

\section{Causa de sangrado mayor}

\section{Procedimiento}

Politraumatismo

Herida por arma blanca

$4(30,76)$

$3(23,07)$

$5(18,39)$

$9(39,13)$

$8(30,76)$

Herida por arma de fuego

$3(23,07)$

$6(26,08)$

$5(19,23)$

Cirugía electiva

$2(15,38)$

$3(13,04)$

$6(23,07)$

Sangrado Ginecobstétrico

$1(7,69)$

$1(3,84)$

$1(4,34)$

$6(23,07)$

Total

$13(100)$

$24(100)$

$26(100)$

$35 \%$

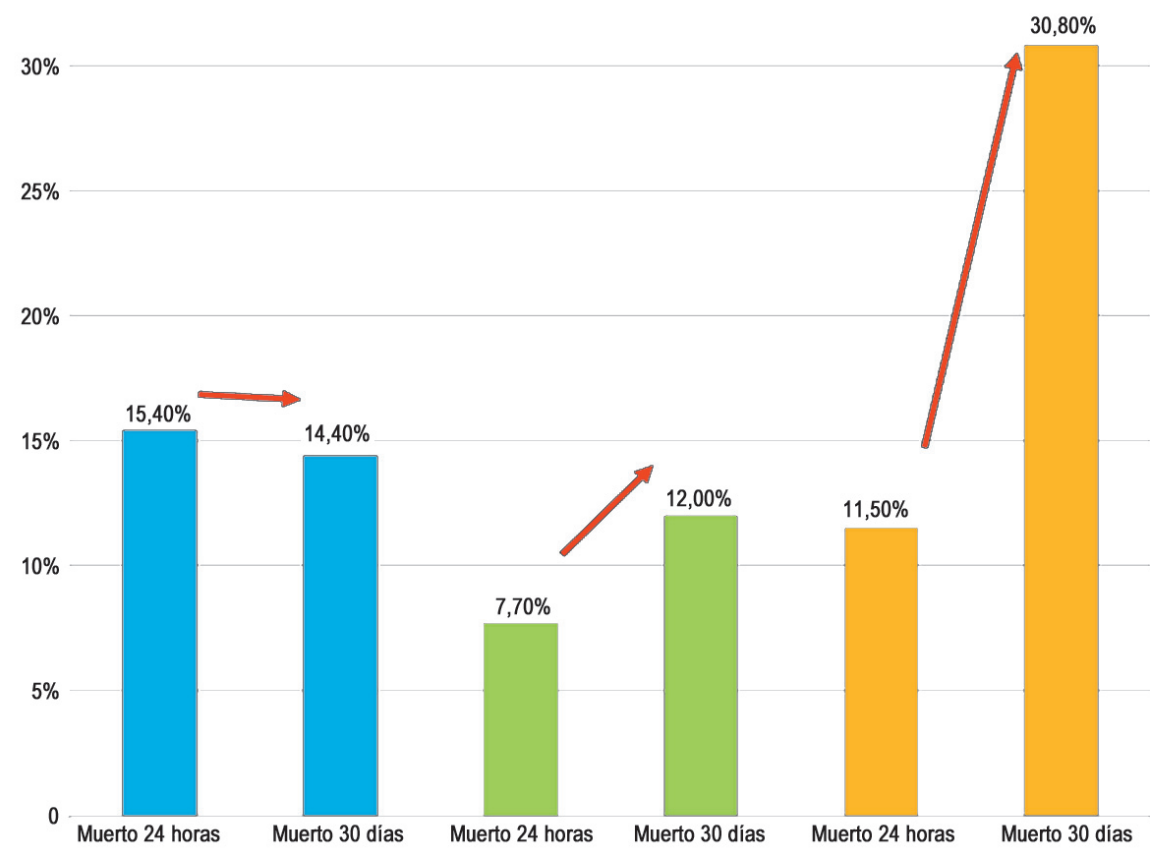

Figura 1. Mortalidad a 24 horas y 30 días según protocolo. 
Tabla 2. Lactato sérico y base exceso a las 24 horas.

\begin{tabular}{|c|c|c|c|c|c|}
\hline Variables No. (\%) & $\begin{array}{l}\text { Protocolo } \\
\text { Completo }\end{array}$ & $\begin{array}{l}\text { Protocolo } \\
\text { Incompleto }\end{array}$ & Control & Total & $\mathbf{P}$ \\
\hline \multicolumn{6}{|c|}{ Lactato sérico a las 24 horas } \\
\hline $\begin{array}{l}\text { Disminuyo } \\
\text { Aumento }\end{array}$ & $\begin{array}{l}6(30,0) \\
9(81,8)\end{array}$ & $\begin{array}{c}1(4,0) \\
14(70,0)\end{array}$ & $\begin{array}{c}2(18,2) \\
24(96,0)\end{array}$ & $\begin{array}{c}9(16,1) \\
47(83,9)\end{array}$ & 0,047 \\
\hline \multicolumn{6}{|c|}{ Base exceso a las 24 horas } \\
\hline $\begin{array}{l}\text { Hacia }(-) \\
\text { Hacia }(+)\end{array}$ & $\begin{array}{c}0 \\
13(100)\end{array}$ & $\begin{array}{l}25(96,2) \\
23(100)\end{array}$ & $\begin{array}{c}0 \\
1(3,8)\end{array}$ & $\begin{array}{l}25(41,0) \\
36(59,0)\end{array}$ & 0,0001 \\
\hline
\end{tabular}

Tabla 3. Unidades de hemoderivados transfundidas en el grupo prospectivo (Tanto protocolo completo como incompleto).

\begin{tabular}{lccccc}
\hline & N & Mínimo & Máximo & Media & Desv. tip. \\
\hline Glóbulos rojos & 36 & 100 & 6,00 & 3,6389 & 0,99003 \\
Plasma & 28 & 3,00 & 8,00 & 4,6786 & 1,21879 \\
Plaquetas & 14 & 3,00 & 6,00 & 4,11429 & 0,66299
\end{tabular}

Fuente: Resultados del estudio.

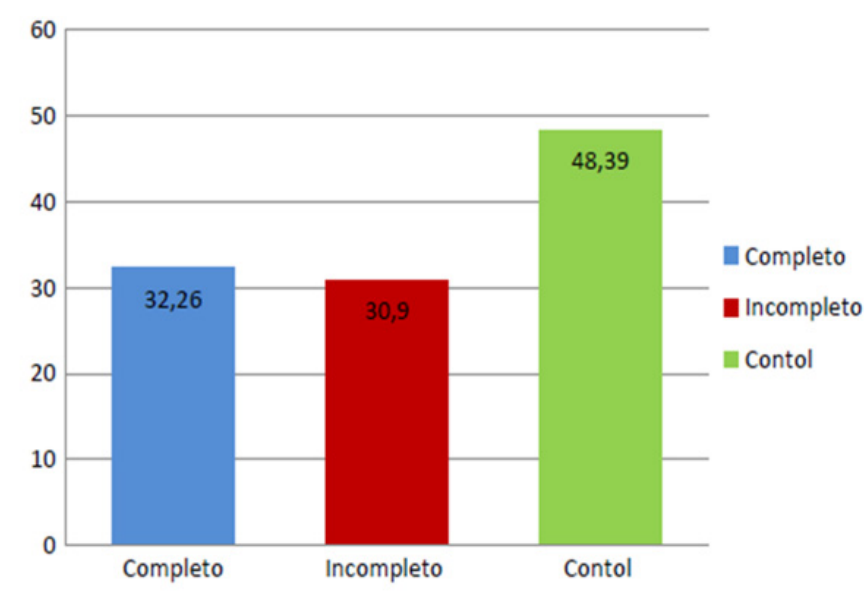

Figura 2. Tasas mortalidad a 24 horas según grupo.

Adherencia al protocolo: Las medidas del protocolo que menos se utilizaron por parte de grupo de anestesiología fue la medición de la temperatura ${ }^{33,34}$, seguido por la relación $1: 1: 1$ de los hemoderivados $(36,11 \%)$.

\section{Discusión}

En Colombia hay una gran relación entre variables culturales, económicas, médicas, sociales y políticas, que influyen de forma directa en la presentación del sangrado mayor ${ }^{26,27}$.
Según el estudio multicéntrico e internacional $\mathrm{CRASH}^{28}$, con participación de hospitales Colombianos, incluyendo el Hospital Universitario de Neiva, de 20.211 pacientes, la mayoría eran hombres, tanto en Colombia 1.512 (90,7\%), como en el resto del mundo, 16.935 (84\%) estimando además que casi el 50\% de las víctimas están entre 15 y 44 años, similar a los datos obtenidos en nuestro estudio, donde la población que más cursa con sangrado mayor son hombres con promedio de edad de 31 años.

En Colombia, según datos obtenidos del Departamento Nacional de Estadísticas DANE ${ }^{29}$ y del Instituto Nacional de Medicina Legal y Ciencias Forenses para el año $2007^{30}$, las lesiones de causa externa se ubicaron en los primeros lugares de las causas de mortalidad general. El homicidio fue la segunda causa después de las lesiones isquémicas cardiacas, las lesiones por accidentes de tránsito y el suicidio ocuparon los lugares 7o y 26o, respectivamente. Esta información también se correlaciona con los datos obtenidos en nuestro estudio en donde las principales causas de sangrado mayor fueron las heridas por arma blanca, las heridas por arma de fuego y las heridas por politraumatismo en accidentes de tránsito.

En Colombia no se cuentan con datos exactos sobre las tasas de mortalidad para aquellos pacientes que cursan con sangrado mayor y son llevados a tratamiento quirúrgico. Nuestro estudio muestra que la tasa de mortalidad a 24 horas varía entre 32 y 48 x 1.000 pacientes. En contraste, la mortalidad a los 30 días se aumenta casi 3 veces (tasa de $129,03 \times 1.000)$ en pacientes sin aplicación del protocolo. El Comité Británico para los Estándares en Hematología de 
R.F.S Revista Facultad de Salud

Enero-Junio de 2015;7(1): 17-23
Evaluación del impacto en la morbimortalidad con la aplicación de un protocolo en sangrado mayor/Daniel Rivera T., Carlos A. Rivera O., Miguel F. Sandoval C.
Julio de 2008, presenta una guía de recomendaciones y estrategias para abordar el sangrado mayor ${ }^{31}$, aun así muchos protocolos y estrategias, algunas empíricas, se han desarrollado para transfundir componentes sanguíneos como uno de los pilares en las estrategias de reanimación frente al sangrado mayor ${ }^{32-38}$. La evidencia científica más reciente basada en las fuerzas militares de los Estados Unidos, enfatizan la administración de glóbulos rojos empaquetados, plasma fresco congelado, y plaquetas en una relación $1: 1: 1$ a $6: 4: 4$ durante las primeras 24 horas pos trauma. Con esta estrategia se ha logrado demostrar una significante disminución de la mortalidad a 24 horas $^{39}$ en comparación con otras formas de realizar la relación en la administración de los componentes sanguíneos.

En nuestro estudio no se contó con la disponibilidad oportuna de hemoderivados, como lo hay en otros centros ${ }^{40}$, y en ocasiones no se cumplió el protocolo al $100 \%$ en el grupo de intervención, por tal razón se conformaron 3 grupos, un grupo control, un grupo al que se le aplicó todo el protocolo y un tercer grupo a quien se le aplicó el protocolo de forma parcial. En nuestro estudio no se tuvo en cuenta el tiempo de almacenamiento de los hemoderivados utilizados ${ }^{41}$ que puede impactar los resultados debido a la lesión por almacenamiento de los hemoderivados ${ }^{42}$.

Consideramos que para lograr resultados estadísticamente significativos se debe ampliar la muestra de pacientes en futuros estudios.

\section{Conclusiones}

- La aplicación de un protocolo estandarizado de manejo disminuye la tasa de Mortalidad de los pacientes con sangrado mayor a las 24 horas y su efecto se magnifica a $\operatorname{los} 30$ días.

- La implementación del protocolo propuesto en este estudio evidencia una mejoría en la morbilidad de los pacientes, el cual se refleja por una disminución a las 24 horas del nivel de lactato sérico y un aumento en los niveles de base exceso.

- Los pacientes que presentan sangrado mayor en el Hospital Universitario de Neiva son en su mayoría hombres en edad promedio de 31 años, donde la causa más común es la herida por arma blanca, sin embargo el politraumatismo ocupa un puesto importante dentro de las causas de sangrado mayor.

- $\quad$ El número de hemoderivados que se deben tener disponible en pacientes con sangrado mayor para que se beneficien con la implementación del protocolo es en promedio de 4 unidades de glóbulos rojos, 4 de unidades de plasma fresco congelado y 4 unidades de plaquetas, manteniendo preferiblemente la relación de 1:1:1 para las transfusiones.

\section{Referencias}

1. PedenM., McGee K., Sharma G. The injury chart book: a graphical overview of the global burden of injuries. Geneva: WorldHealthOrganization; 2002.
2. Forero L., González J., Bohórquez G., Carreño P., InsuastyJ., Soriano M. Datos Para La Vida. Instituto Nacional De Medicina Legal Y Ciencias Forenses. Bogotá, Colombia: Forensis; 2007.

3. Juan A. Asensio, Esther Rojo, PatrizioPetrone. Síndrome de exanguinación. Factores predictivos e indicativos para la institución de la cirugía de control de daños. Circular Española 2003;73(2):120-9

4. Cinat ME, Wallace WC, Nastanski F, et al. Improved survival following massive transfusion in patients who have undergone trauma. Arch Surg. 1999;134:964- 968.

5. KarimBrohia, Mitchell J. Cohenband Ross A. Acute coagulopathy of trauma: mechanism, identification and effect. Curr Opin Crit Care 2007. 13:680-685.

6. KauvarDs.,LeferingR., Wade Ce. Impact Of Hemorrhage On Trauma Outcome: An Overview Of Epidemiology, Clinical Presentations, And Therapeutic Considerations. J Trauma. 2006;60(Suppl.):S3-11.

7. HeikoLier, MD, Henning Krep, MD, PhD, Stefan Schroeder, MD, PhD, and Frank Stuber, MD, PhD. Preconditions of Hemostasis in Trauma: A Review. The Influence of Acidosis, Hypocalcemia, Anemia, and Hypothermia on Functional Hemostasis in Trauma. The Journal of TRAUMA Injury, Infection, and Critical Care. J Trauma. 2008;65:951-960.

8. Kristen C. Sihler, MD, MS; And Lena M. Napolitano, MD. Complications Of Massive Transfusion. 2010 American College Of Chest Physicians. Chest/137/1/ January, 2010.

9. Jeffry L. Kashuk, MD, Ernest E. Moore, MD, Michael Sawyer, MD, Tuan Le, MD, Jeffrey Johnson, MD, Walter L. Biffl, MD, C. Clay Cothren, MD, Carlton Barnett, MD, Philip Stahel, MD, Christopher C. Sillman, MD, PhD, Angela Sauaia, MD, PhD, and Anirban Banerjee, PhD. Postinjury Coagulopathy Management Goal Directed Resuscitation via POC Thrombelastography. Annals of Surgery, Volume 251, Number 4, April 2010. (Ann Surg 2010;251:604-614).

10. Fernando Raffán Sanabria1, Francisco J. Ramírez P2, Juan Andrés Cuervo, Lina F. Sánchez Marí. Tromboelastografía. Revista Colombiana de Anestesiología. Rev. Col. Anest. vol.33 no.3 Bogotá July/Sept. 2005

11. Maegele $M$, Lefering $R$, Paffrath $T$, et al. Red-blood-cell to plasma ratios transfused during massive transfusion are associated with mortality in severe multiple injury: a retrospective analysis from the Trauma Registry of the Deutsche Gesellschaft fur Unfallchirurgie. Vox Sang. 2008;95:112-119.

12. Sperry JL, Ochoa JB, Gunn SR, et al. An FFP: PRBC transfusion ratio is associated with lower risk mortality after massive transfusion. J Trauma. 2008;65:986-993.

13. HeikoLier, MD, H. Krep, MD, PhD, Stefan Schroeder, MD, PhD, and Frank Stuber, MD, PhD. Preconditions of Hemostasis in Trauma: A Review. The Influence of Acidosis, Hypocalcemia, Anemia, and Hypothermia on Functional Hemostasis in Trauma. The Journal of Traumalnjury, Infection, and Critical Care. Volume 65 • 
Evaluación del impacto en la morbimortalidad con la aplicación de un protocolo en sangrado mayor/Daniel Rivera T., Carlos A. Rivera O., Miguel F. Sandoval C.
R.F.S Revista Facultad de Salud

Enero-Junio de 2015;7(1): 17-23
Number 4. DOI: 10.1097/TA.0b013e318187e15b. AcceptedforpublicationJuly 24, 2008.

14. D Stainsby, S MacLennan, D Thomas, J Isaac. Additional consultation and advice: PJ Hamilton. Guidelines on the Management of Massive Blood Loss. British Committee for Standards in Haematology.July 2008. bcsh@b-s-h.org.uk.

15. American College of Surgeons Committee on Trauma. Advanced Trauma Life Support Instructor Manual. Chicago: American College of Surgeons, 1997.

16. Gubler KD, Gentilello LM, Hassantash SA, Maier RV. The impact of hypothermia on dilutional coagulopathy. J Trauma. 1994;36:847-851.

17. Philip F. Stahela, Ernest E. Mooreb, Star L. Schreiera, Michael A. Flierlaand Jeffry L. Kashukb. Transfusion strategies in postinjury coagulopathy. CurrentOpinion in Anaesthesiology 2009;22:289-298.

18. Borgman $M$, Spinella $P$, Perkins $M$, et al. The ratio of blood products transfused affects mortality in patients receiving massive transfusions at a combat support hospital. J Trauma. 2007;63:805-813.

19. Janis Bormanis. Development of a massive transfusion protocol. Transfusion and Apheresis Science 38 (2008) 57-63.

20. Delgado A, Dong J, Chambers J, et al. Effects of hypothermia and acidosis on swine platelet activation and aggregation in vivo. J ThrombHaemost. 2005; 3(suppl 1):Abstract number P2084 from the XXth ISTH congress, 6-12th August 2005.

21. Martini WZ, Dubick MA, Pusateri AE, Park MS, Ryan KL, Holcomb JB. Does bicarbonate correct coagulation function impaired by acidosis in swine? J Trauma. 2006;61:99-106.

22. Broder G, Weil MH: Excess lactate: an index of reversibility of shock in human patients. Science 1964;143:14571459.

23. Spahn DR. Hypocalcemia in trauma: frequent but frequently undetected and underestimated. Editorial. CritCareMed. 2005; 33:2124-2125.

24. Suzuki N, Fujimoto Z, Morita T, Fukamizu A, Mizuno $H$. $\mathrm{pH}$ - Dependent structural changes at $\mathrm{Ca}(2)$-binding sites of coagulation factor IX binding protein. J Mol Biol. 2005;353:80 - 87 .

25. Jorge Humberto Mejía Mantilla, FabianEduardo Puentes-Manosalva, Juan Diego Ciro, Carlos Morales. Hemorragia Y Trauma, Avances Del Estudio Crash2 En Colombia. Revista Colombiana De Cirugía. 2009; 24:175-83.

26. Informe OPS 2008 - Instituto Nacional de Salud Coordinación http://www.ins.gov.co/index.php?id categoria $=9293$.

27. Informe OPS 2007 - Instituto Nacional de Salud - Coordinación Nacional de Sangre. Disponible en http:/ /www.ins.gov.co/index.php?idcategoria $=9317$

28. CRASH-2 trial collaborators. Clinical Trials Unit, London School of Hygiene and Tropical Medicine. E? ects of tranexamic acid on death, vascular occlusive events, and blood transfusion in trauma patients with signi? cant haemorrhage (CRASH-2): a randomised, placebo- controlled trial. Published Online June 15, 2010. DOI:10. 1016/S0140-6736(10)60835-5.crash@lshtm.ac.uk

29. Departamento Administrativo Nacional de Estadística de Colombia DANE. http://buscador.dane.gov.co/search? $q=$ trauma + en +colombia $+a \% C 3 \% B 1 o+2007 \& b$ tnG.x $=$

30 Instituto Nacional de Medicina Legal y Ciencias forense de Colombia. http://www.medicinalegal.gov.co/index.

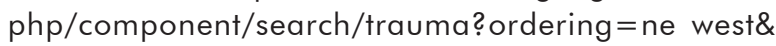
searchphrase $=$ all\&limit $=100 \#$ content.

31. D Stainsby, S MacLennan, D Thomas, J Isaac. Additional consultation and advice: PJ Hamilton. Guidelines on the Management of Massive Blood Loss. British Committee for Standards in Haematology.July 2008. bcsh@b-sh.org.uk.

32. Pier MannuccioMannucci, M.D., and Marcel Levi, M.D., Ph.D. Prevention and Treatment of Major Blood Loss. Thenew england journal of medicine. $2007 ; 356$ : 2301-11.

33. KenrickBerend, MD, PhD,Marcel Levi, MD, PhDManagement of Adult Jehovah's Witness Patients wit Acute Bleeding. The American Journal of Medicine (2009) 122, 1071-1076.

34. Donat R Spahn, Vladimir Cerny, Timothy J Coats, Jacques Duranteau, Enrique Fernández- Mondéjar, Giovanni Gordini, Philip F Stahel7, Beverley J Hunt, RadkoKomadina, Edmund Neugebauer, Yves Ozier, Louis Riddez, Arthur Schultz, Jean-Louis Vincentand Rolf Rossaint. Management of bleeding following major trauma: a European guideline Published: 13 Feb 2007 Critical Care 2007, 11:R17 (doi:10.1186/cc5686) This article is online at: http://ccforum.com/content/1 1/1/R17

35. S. Kozek-Langenecker. Management Of Massive Operative Blood Loss. Minerva Anestesiologica. 2007 ; 73(7-8):401-15.

36. Rossaint R, Cerny V, Coats TJ, et al. Key issues in advanced bleeding care in trauma. Shock. 2006; 26:322-331.

37. Rivera T.D., Pérez F.A. Artículo de Revisión "Técnicas de Ahorro Sanguíneo en Cirugía". Rev Col Anest 2011, Vol. 39 No. 4: pág. 545-559.

38. Kerstin S Schick, Jan M Fertmann, Karl-Walter Jauch and Johannes $N$ Hoffmann. Prothrombin complex concentrate in surgical patients:retrospective evaluation of vitamin $\mathrm{K}$ antagonist reversal and treatment of severe bleeding. CriticalCare 2009, 13:R191 (doi:10.1186/cc8186)

39. Snyder CW, Weinberg JA, Mcgwin G, et al. The relationship of blood product ratio to mortality: survival benefit or survival bias? J Trauma. 2009;66:358 -364.

40. Spahn DR, Cerny V, Coats TJ, et al. Management of bleeding following major trauma: a European guideline. Crit Care. 2007;11:R17.

41. Raat NJ, Berends F, Verhoeven AJ, de Korte D, Ince C. The age of stored red blood cell concentrates at the time of transfusion. TransfusMed. 2005;15:419-423.

42. Rivera T.D. Almacenamiento sanguíneo y transfusión. Rev Colomb Anestesiol. 2013;41:75 - Vol. 41 Núm.01 DOI: $10.1016 /$ i.rca.2012.08.005. 